\title{
Patient adherence to the face-down positioning after macular hole surgery
}

This article was published in the following Dove Press journal:

Clinical Ophthalmology

8 June 2017

Number of times this article has been viewed

\section{Yoshiaki Shimada \\ Yui Seno \\ Tadashi Mizuguchi \\ Atsuhiro Tanikawa \\ Masayuki Horiguchi}

Department of Ophthalmology, Fujita Health University Hospital, Aichi, Japan
Correspondence: Yoshiaki Shimada Department of Ophthalmology, Fujita Health University Hospital, I-98 Dengakugakubo, Kutsukake-cho, Toyoake, Aichi 470-I 192, Japan

Tel +8I 562932000

Email ysmd@fujita-hu.ac.jp
Purpose: To determine adherence to face-down positioning (FDP) among patients who underwent vitrectomy and gas tamponade for macular hole (MH) repair.

Method: A total of 69 patients ( 37 females and 32 males) who underwent primary vitrectomy for MH repair were studied. Nurses recorded whether the patient complied with FDP each time they examined the patient. FDP score was obtained from the nursing records; patients were given a score of 1 if they complied with the FDP and 0 if they did not. The score was recorded four times per day for the first 3 postoperative days. A perfect FDP score was 12 .

Results: The mean \pm standard deviation of the FDP scores was 10.6 \pm 1.8 (range: 4-12). Overall, $32(46.4 \%)$ patients scored a perfect 12 , and $7(10.1 \%)$ patients scored $<8$. Failure of the MH closure was observed in only one patient $(1.4 \%$ ), who showed the poorest adherence to FDP (score $=4$ ). Consequently, the closure rate in patients with FDP score $<7(2 / 3,66.7 \%)$ was significantly lower than in patients with an FDP score $\geq 7(66 / 66,100 \%)(P<0.05$, Fisher's exact probability test). Conclusion: While adherence to FDP after MH surgery was better than that observed after vitrectomy for rhegmatogenous retinal detachments in our previous study, the percentage of patients with FDP scores $<8$ did not differ. Statistically, the poor adherence to FDP can negatively impact the effectiveness of the surgery for MH repair.

Keywords: adherence, face-down positioning, gas tamponade, macular hole, vitrectomy

\section{Introduction}

Face-down positioning (FDP) is a standard posture recommended for patients following vitrectomy and gas tamponade procedures during macular hole $(\mathrm{MH})$ surgery. ${ }^{1-28}$ Patients find the FDP inconvenient, and so it is not readily tolerated; therefore, the optimal method and duration of the FDP have been debated for many years. ${ }^{2-28}$ Shortening the duration of the FDP $2,5,8,10,11,14,16,17,22$ and adopting alleviated positioning, such as avoidance of the supine or face-up positioning by the patients, ${ }^{6-13,15,19,20,22-25,27,28}$ have been proposed and statistically compared with strict FDP. ${ }^{5,7-12,21-25,27,28}$ However, these studies lacked adherence controls, and the intervention was the advice given to the patients to maintain the positioning rather than the positioning itself. ${ }^{21}$ Even if the same advice was given to all the patients, some patients complied strictly, whereas others did not. Therefore, the disclosure of the actual adherence scores will help access the necessity of FDP in the prognosis of MH surgery.

Recently, we investigated 127 patients who underwent vitrectomy for rhegmatogenous retinal detachment. ${ }^{29}$ We observed that the adherence to FDP varied considerably among the patients and that some patients failed to maintain the FDP for nearly or more than half the prescribed the time. The purpose of this study was to determine the differences in adherence to FDP in patients with causative diseases and the impact of adherence on the prognosis after $\mathrm{MH}$ surgery. 


\section{Method}

\section{Methodology and subjects}

We retrospectively examined the nursing records of patients who had undergone primary vitrectomy and gas tamponade for idiopathic MH at the Fujita Health University Hospital (Toyoake, Japan) for a period of 1 year between April 2012 and March 2013. A total of 69 patients (37 females and 32 males) were included. The nursing records included detailed information related to the adherence of the patients to FDP.

\section{Surgery}

All the patients who had signed consent forms for the surgery and FDP received instructions on FDP. Pars plana vitrectomy was performed by experienced surgeons. Further, each patient also underwent triamcinolone-assisted internal limiting membrane peeling of approximately two disc diameters, prophylactic phacoemulsification, intraocular lens implantation, a complete fluid-gas exchange, and gas tamponade with $20 \%$ sulfur hexafluoride.

Postoperatively, the patients were advised to maintain FDP as much as possible every day for at least $1 \mathrm{wk}$. After the second week, when the MH closure was confirmed by optical coherence tomography, some patients were advised only to avoid the face-up position until the disappearance of the intraocular gas.

\section{Nursing records}

If the patients underwent gas tamponade, this was indicated in the hospital chart. Each time the patient was examined, including the four routine ward rounds per day, the nurse recorded whether the patient had complied with FDP. The nurse instructed the patient to resume FDP. If patients were found sleeping in a position other than FDP, the nurse woke them and asked them to assume FDP.

Each time the patient was checked, the nurse entered the data regarding the patient adherence to FDP into a handheld terminal. The data were exported and stored in a digital hospital chart.

\section{FDP Score}

To quantify the patients' adherence to FDP, the position of each patient was scored four times per day: at midnight, morning, midday, and evening, at approximately $24: 00 \mathrm{~h}$, 6:00 h, 12:00 h, and 18:00 h, respectively. Patient monitoring began at 24:00 $\mathrm{h}$ on the day of the surgery. Although the nurses continued these examinations until the gas disappeared or the patient was discharged, we only counted the score for the first 3 consecutive days in our initial attempt. ${ }^{29}$
Consequently, a total of 12 observations were recorded, and the patients were given a score of 1 each time they followed FDP. Thus, a patient with perfect adherence would have a score of 12 (Figure 1).

\section{Results}

\section{Outline and gender differences}

Table 1 shows the demographics of all the patients. The mean \pm standard deviation of the FDP score was $10.6 \pm 1.8$ (range, 4-12) for all the patients. The mean FDP score in female patients $(10.9 \pm 1.5)$ was higher than that in males (10.3 \pm 2.1$)$; however, this difference was not statistically significant $(P>0.2$, Mann-Whitney $U$ test).

A total of 32 patients $(46.4 \%)$ had a perfect score of 12 , which included 19 (51.4\%) females and 13 (40.6\%) males. Seven patients $(10.1 \%)$ were given low scores, ${ }^{29}$ with an FDP score $<8$, including two females $(5.4 \%)$ and five males $(15.6 \%)$. There were more perfect scorers and fewer low scorers among female patients than that among male patients; however, there were no statistically significant differences between the males and females $(P>0.37$ and $P>0.16$, respectively, $\chi^{2}$ test).

\section{Age analysis and individual observations}

Figure 2 shows the distribution of the FDP score as a function of age. No significant correlation of the FDP score with age was observed, irrespective of sex.

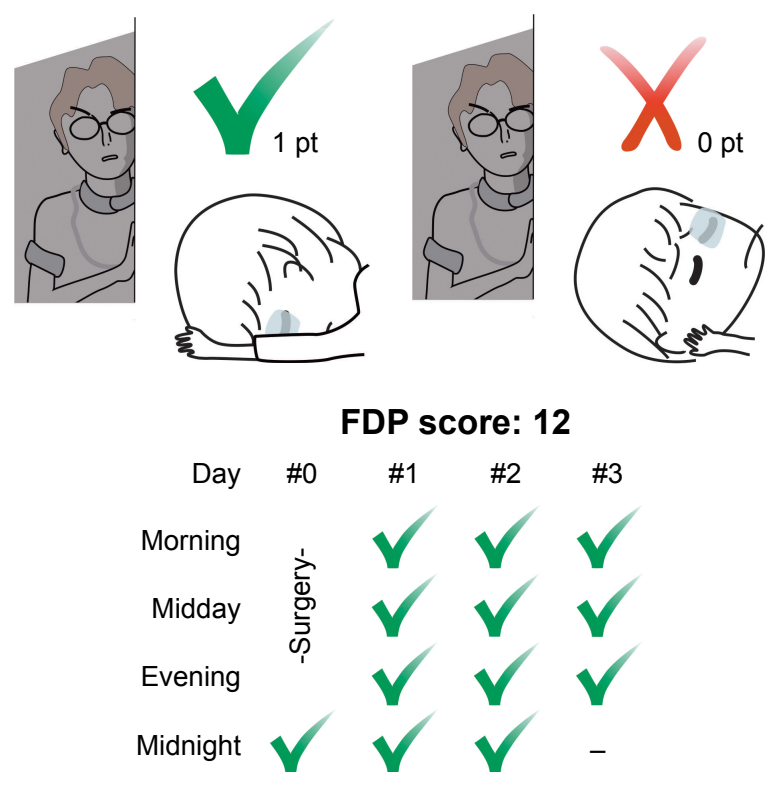

Figure I FDP score.

Notes: Patients were given I point every time they followed FDP (top left panel); otherwise, the score was 0 (top right panel). Scores were recorded four times per day, from midnight on the day of the surgery up to 3 consecutive days: midnight $(24: 00 \mathrm{~h})$, morning $(6: 00 \mathrm{~h})$, midday (12:00 h), and evening (18:00 h). A perfect FDP score was 12 (bottom panel).

Abbreviation: FDP, face-down positioning. 
Table I Subjects and the FDP score

\begin{tabular}{llll}
\hline & \multirow{2}{*}{$\begin{array}{l}\text { All } \\
\text { subjects }\end{array}$} & \multicolumn{2}{l}{ Gender comparison } \\
\cline { 3 - 4 } & & Female & Male \\
\hline $\mathrm{n}$ (cases) & 69 & 37 & 32 \\
Eye (cases, right/left) & $40 / 29$ & $24 / 13$ & $16 / 16$ \\
$\begin{array}{l}\text { Age (years, mean } \pm \text { SD) } \\
\text { FDP score }\end{array}$ & $66.2 \pm 6.8$ & $66.0 \pm 6.8$ & $66.4 \pm 6.9$ \\
$\quad$ & & & \\
$\quad$ (mean \pm SD) & $10.6 \pm 1.8$ & $10.9 \pm 1.5$ & $10.3 \pm 2.1$ \\
Less than 8 (cases) & $32(46.4 \%)$ & $19(51.4 \%)$ & $13(40.6 \%)$ \\
\hline
\end{tabular}

Abbreviations: FDP, face-down positioning; SD, standard deviation.

Patients who failed to score a perfect 12 FDP score included a 79-year-old male (\#10) with a score of 9 and a 64-year-old female (\#14) with a score of 10 . The lowest scorers included a 72-year-old male (\#26) with a score of 6 and a 55-year-old male (\#48) with a score of 4 (Figure 3). As the patient did not follow FDP, the data regarding their actual body position are not available; however, there was some data available, for example, the patients often remained in the supine position or sitting upright position during the daytime hours.

Patients \#10 and \#14 had intermediate scores, which are shown in the upper panels of Figure 3. These patients usually maintained FDP during the day, but tended to lose a point at midnight. The patients who showed the poorest adherence (\#26 and \#48) did not maintain FDP even during the day (lower panels of Figure 3). Any disability or any problematic behavior was not mentioned in the nursing records. The patients understood the advice given by the nurse each time but were often found in the supine position during the next examination. Moreover, repeated instructions did not improve their adherence.

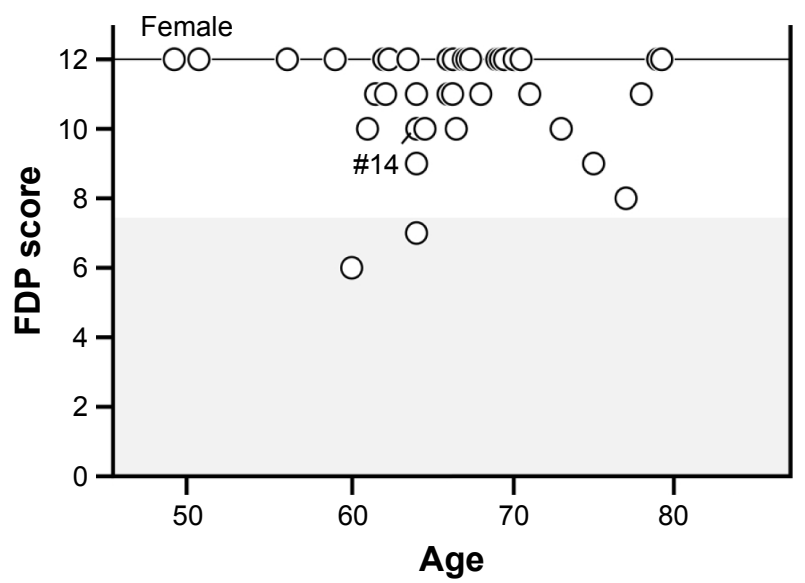

\section{Prognosis after surgery}

Primary anatomical MH closure was achieved in 68 eyes (98.6\%). Only one patient (1.4\%, \#48) failed to achieve $\mathrm{MH}$ closure, and this patient had the lowest FDP score (Figures 2 and 3). Consequently, the closure rate in patients with an FDP score $<8(6 / 7,85.7 \%)$ was lower than that in patients with an FDP score $\geq 8(62 / 62,100 \%)$; however, the difference was not statistically significant $(P<0.11$, Fisher's exact probability test). A statistically significant difference was observed when a FDP score of 7 was used as the cutoff level; the closure rate in patients with FDP score $<7(2 / 3$, $66.7 \%$ ) was significantly lower than that in patients with an FDP score $\geq 7$ (66/66, 100\%) (Table 2, $P<0.05$, Fisher's exact probability test).

On preoperative assessment of patient \#48, the $\mathrm{MH}$ was $390 \mu \mathrm{m}$ in diameter and consistent with Gass stage 3 . He underwent a second surgery 10 days after the first surgery. Complete fluid-gas exchange and gas tamponade with $20 \%$ sulfur hexafluoride were performed again without repeating the removal of internal limiting membrane. This patient's FDP score after the second surgery was a perfect 12, and his MH closed completely.

Another patient, $\# 26$, who underwent a repeat surgery, was also one of the low scorers (Figures 2 and 3). His MH closed successfully; however, he developed a rhegmatogenous retinal detachment in the same eye 6 months later. During the second vitrectomy procedure, the causative retinal break was closed and the retina was reattached. The FDP score after the second surgery was 9, whereas it was 6 after the first surgery. The relationship between poor adherence after the first surgery and the onset of retinal detachment was not determined.

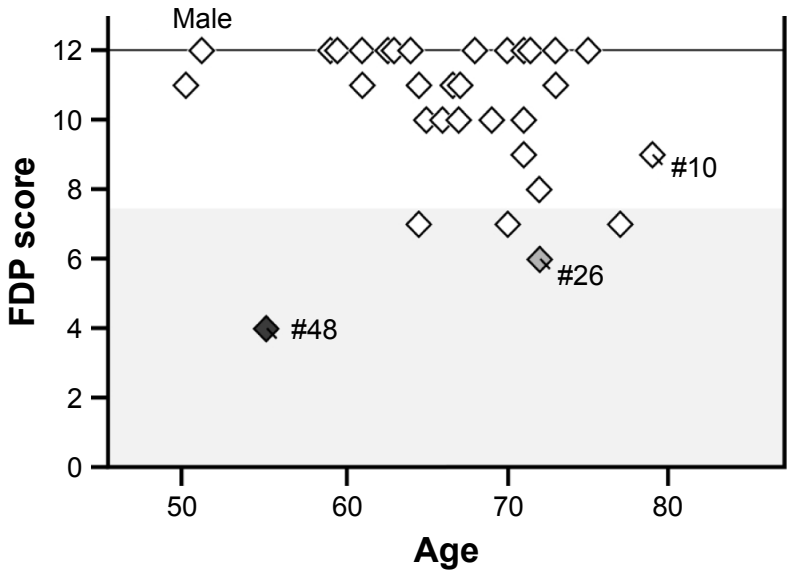

Figure 2 Age distribution of the FDP score in females (left panel) and in male patients (right panel).

Notes: The filled tetragon in the bottom panel represents a 55-year-old male (\#48), who was the only patient who failed to achieve initial MH closure. The shaded tetragon in the bottom panel represents a 72-year-old male (\#26) who developed a rhegmatogenous retinal detachment in the same eye 6 months later.

Abbreviations: FDP, face-down positioning; $\mathrm{MH}$, macular hole. 


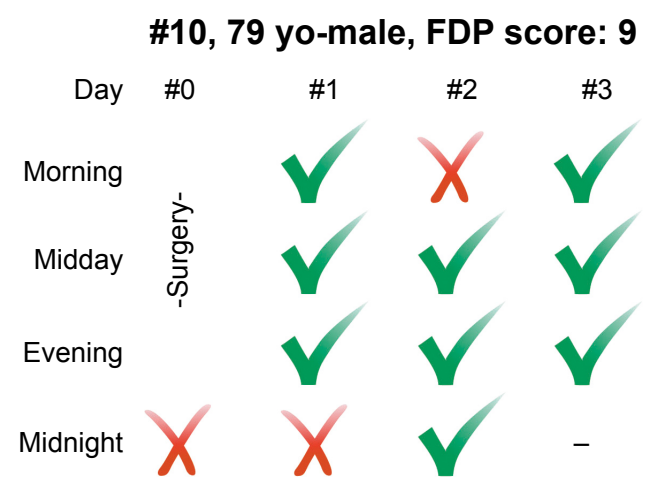

\#26, 72 yo-male, FDP score: 6

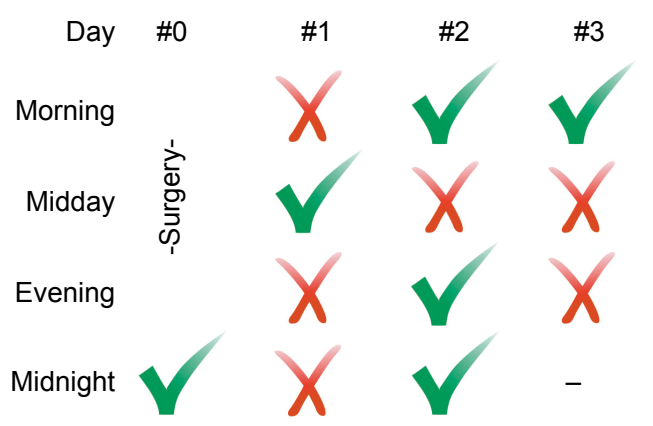

$\# 14,64$ yo-female, FDP score: 10

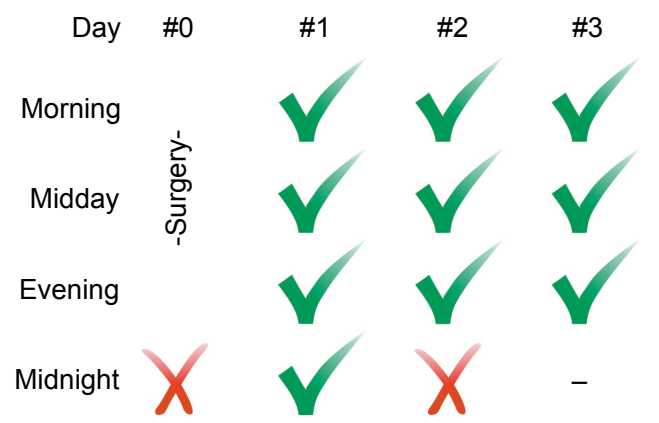

\#48, 55 yo-male, FDP score: 4

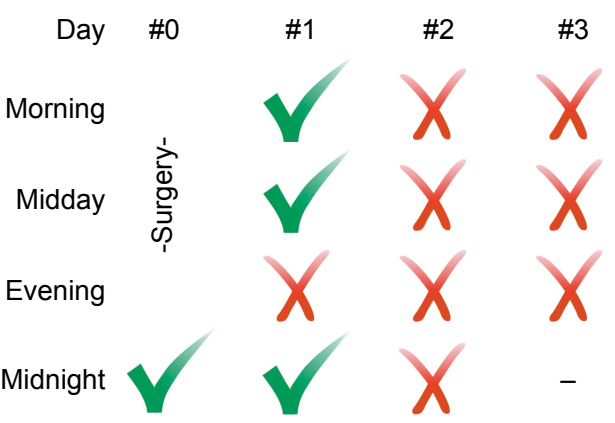

Figure 3 FDP score of patients who failed to score a perfect 12: \#10 represents a 79-year-old male with an FDP score of 9 (top left panel); \#I4 represents a 64 -year-old female with an FDP score of 10 (top right panel); \#26 represents a 72-year-old male with an FDP score of 6 (bottom left panel); and \#48 represents a 55 -year-old male with an FDP score of 4 (bottom right panel).

Abbreviations: FDP, face-down positioning; yo, year old.

\section{Discussion}

Mechanical sensory devices have been produced and tested in two pilot studies involving $10^{4}$ and $13^{21}$ patients, respectively, to assess the posture of the head after $\mathrm{MH}$ surgery. Although the device recorded the patient's head position continuously for $24 \mathrm{~h}$, it had to be mounted on the patient's head, which could increase the strain on the patients. Although the FDP score measured by us is a rough index obtained only from the nursing records, it can still be retrospectively used for the evaluation of many patients and their surgical outcomes. ${ }^{29}$ In this study, approximately $50 \%$ of the females and $40 \%$ of the males had a perfect FDP score of 12. However, the sampling frequency of four times per day was not sufficient to actually determine whether their

Table 2 Closure rate

\begin{tabular}{|c|c|c|c|c|c|}
\hline & \multirow{2}{*}{$\begin{array}{l}\text { All } \\
\text { subjects }\end{array}$} & \multicolumn{4}{|c|}{ FDP score } \\
\hline & & $\geq 7$ & & $<7$ & \\
\hline $\mathrm{n}$ (cases) & 69 & 66 & & 3 & \\
\hline $\begin{array}{l}\text { Primary } \\
\text { closure (cases) }\end{array}$ & 68 (98.6\%) & $66(100 \%)$ & $* 1$ & 2 (66.7\%) & $* 1$ \\
\hline
\end{tabular}

Note: *I represents $P<0.05$ Fisher's exact probability test. Abbreviation: FDP, face-down positioning. adherence was perfect; nonetheless, we can assume that they showed good adherence.

Patients who did not have a perfect score were often not found in FDP at midnight, which may possibly be attributable to deep sleep. Further, this was also found to be the case in a study which used a head-mounted monitoring device. ${ }^{4}$ The effort required to sleep in FDP may be different from that required to remain in FDP during the awake hours, and this may be a key determination of the degree of adherence for many patients.

Low scorers, who failed nearly or more than half the time, did not maintain FDP even during the day. It should be noted that the patients surveyed in this research were kept in the hospital under observation. If they had recovered at home after the surgery, their adherence would likely have been worse.

In an analysis of the FDP scores in 127 patients after vitrectomy for rhegmatogenous retinal detachments at the same facility over the same time period, $31.1 \%$ of females and $29.3 \%$ of males were perfect scorers, ${ }^{29}$ which is lower than that observed in the present study. This may be because of the urgency of surgery for retinal detachments. In cases of retinal detachments, many patients underwent surgery on the first day of their hospital visit. However, patients 
with $\mathrm{MH}$ had a substantial amount of time to prepare for their surgery because their surgery was scheduled at least 1 month in advance. Consideration time for surgery and FDP could improve patient adherence. However, the percentage of patients with FDP scores $<8$ did not differ; the overall rate among patients with retinal detachment was $9.4 \%$ and that among males was $14.6 \% .{ }^{29}$ This indicates that even the long preparation period had little effect on the patients who showed the poorest adherence.

In this study, the lowest scorer was the only patient who failed to achieve the initial MH closure. Statistically, it suggests that poor adherence to FDP was responsible for the failed MH surgery. After a second surgery, this patient's adherence drastically improved and the $\mathrm{MH}$ closure was successfully achieved.

In the treatment of $\mathrm{MH}$, the optimal manner and the duration of the FDP to optimize patient recovery have been argued. ${ }^{2-28}$ Although each protocol assumes that the patients will follow the given advice, some patients (approximately $10 \%$ ) do not comply. Although they may appear compliant, their poor prognosis can negatively impact the effectiveness of the surgery.

\section{Conclusion}

Adherence may be improved by various measures, for example, by supplying an inflatable assistant device ${ }^{18}$ or fastening a tennis ball to the back of the patient's nightshirt to keep the patient in a nonsupine position. ${ }^{19}$ Poor adherence could be attributed to the inconvenience of FDP; therefore, alleviated positioning, 6-13,15,19,20,22-25,27,28 which may be easier to comply with, may improve patient adherence and postoperative outcomes.

\section{Ethics approval and consent to participate}

This study adhered to the tenets of the Helsinki Declaration and was approved by the Institutional Review Board of Fujita Health University (IRB no 16-036). For this type of study, hospital-based and retrospective, formal consent is not required. IRB of Fujita Health University waived the patient written informed consent for this study as patient data confidentiality was maintained at all times.

\section{Acknowledgments}

The dataset supporting the findings of this study is available upon request from Yoshiaki Shimada, MD (e-mail: ysmd@ fujita-hu.ac.jp). A part of this study was presented at the 8th Asia Pacific Vitreo-retina Society Congress 2013.12.7, Nagoya, Japan, as a poster presentation.

\section{Author contributions}

Shimada drafted the manuscript; Seno and Mizuguchi were responsible for data acquisition; Tanikawa and Horiguchi performed the surgery. All authors contributed toward data analysis, drafting and critically revising the paper and agree to be accountable for all aspects of the work.

\section{Disclosure}

The authors report no other conflicts of interests in this work.

\section{References}

1. Kelly NE, Wendel RT. Vitreous surgery for idiopathic macular holes results of a pilot Study. Arch Ophthalmol. 1991;109:654-659.

2. Thompson JT, Smiddy WE, Glaser BM, Sjaarda RN, Flynn HW Jr. Intraocular tamponade duration and success of macular hole surgery. Retina. 1996;16:373-382.

3. Tornambe PE, Poliner LS, Grote K. Macular hole surgery without face-down positioning. A pilot study. Retina. 1997;17:179-185.

4. Verma D, Jalabi MW, Watts WG, Naylor G. Evaluation of posturing in macular hole surgery. Eye (Lond). 2002;16:701-704.

5. Krohn J. Duration of face-down positioning after macular hole surgery: a comparison between 1 week and 3 days. Acta Ophthalmol Scand. 2005;83:289-292.

6. Dhawahir-Scala FE, Maino A, Saha K, Mokashi AA, McLauchlan R, Charles S. To posture or not to posture after macular hole surgery. Retina. 2008;28:60-65.

7. Guillaubey A, Malvitte L, Lafontaine PO, et al. Comparison of facedown and seated position after idiopathic macular hole surgery: a randomized clinical trial. Am J Ophthalmol. 2008;146:128-134.

8. Tatham A, Banerjee S. Face-down posturing after macular hole surgery: a meta-analysis. Br J Ophthalmol. 2010;94:626-631.

9. Tadayoni R, Vicaut E, Devin F, et al. A randomized controlled trial of alleviated positioning after small macular hole surgery. Ophthalmology. 2011;118:150-155.

10. Chandra A, Charteris DG, Yorston D. Posturing after macular hole surgery: a review. Ophthalmologica. 2011;226(Suppl 1):3-9.

11. Solebo AL, Lange CA, Bunce C, Bainbridge JW. Face-down positioning or posturing after macular hole surgery. Cochrane Database Syst Rev. 2011;(12):CD008228.

12. Lange CA, Membrey L, Ahmad N, et al. Pilot randomised controlled trial of face-down positioning following macular hole surgery. Eye (Lond). 2012;26:272-277.

13. Yagi F, Takagi S, Tomita G. Combined idiopathic macular hole vitrectomy with phacoemulsification without face-down positioning. J Ophthalmol. 2012;2012:571748.

14. Almeida DR, Wong J, Belliveau M, Rayat J, Gale J. Anatomical and visual outcomes of macular hole surgery with short-duration 3-day face-down positioning. Retina. 2012;32:506-510.

15. Nadal J, Delas B, Piñero A. Vitrectomy without face-down posturing for idiopathic macular holes. Retina. 2012;32:918-921.

16. Malik A, Dooley I, Mahmood U. Single night postoperative prone posturing in idiopathic macular hole surgery. Eur J Ophthalmol. 2012;22:456-460

17. Xirou T, Theodossiadis PG, Apostolopoulos M, et al. Macular hole surgery with short-acting gas and short-duration face-down positioning. Clin Ophthalmol. 2012;6:1107-1112.

18. Schaefer H, Koss MJ, Singh P, Koch F. Significant improvement in compliance with the face-down position after vitrectomy and gas tamponade. Klin Monatsbl Augenheilkd. 2012;229:928-936.

19. Forsaa VA, Raeder S, Hashemi LT, Krohn J. Short-term postoperative non-supine positioning versus strict face-down positioning in macular hole surgery. Acta Ophthalmol. 2013;91:547-551. 
20. Iezzi R, Kapoor KG. No face-down positioning and broad internal limiting membrane peeling in the surgical repair of idiopathic macular holes. Ophthalmology. 2013;120:1998-2003.

21. Leitritz MA, Ziemssen F, Voykov B, Bartz-Schmidt KU. Usability of a gravity- and tilt-compensated sensor with data logging function to measure posturing compliance in patients after macular hole surgery: a pilot study. Graefes Arch Clin Exp Ophthalmol. 2014;252:739-744.

22. Heffez JL. Macular hole repair without face-down positioning. Int Ophthalmol Clin. 2014;54:1-15.

23. Feist RM Jr, Pomerleau DL, Feist R, et al. Nonsupine positioning is preferred by patients over face-down positioning and provides an equivalent closure rate in 25- and 23-gauge macular hole surgery. Retin Cases Brief Rep. 2014;8:205-208.

24. Alberti M, la Cour M. Face-down positioning versus non-supine positioning in macular hole surgery. Br J Ophthalmol. 2015;99:236-239.

25. Pasu S, Bunce C, Hooper R, Thomson A, Bainbridge J. PIMS (Positioning In Macular hole Surgery) trial - a multicentre interventional comparative randomised controlled clinical trial comparing face-down positioning, with an inactive face-forward position on the outcome of surgery for large macular holes: study protocol for a randomised controlled trial. Trials. 2015;16:527.
26. Essex RW, Kingston ZS, Moreno-Betancur M, et al. The effect of postoperative face-down positioning and of long- versus shortacting gas in macular hole surgery: results of a registry-based study. Ophthalmology. 2016;123:1129-1136.

27. Hu Z, Xie P, Ding Y, Zheng X, Yuan D, Liu Q. Face-down or no facedown posturing following macular hole surgery: a meta-analysis. Acta Ophthalmol. 2016;94:326-333.

28. Alberti M, la Cour M. Nonsupine positioning in macular hole surgery: A Noninferiority Randomized Clinical Trial. Retina. 2016;36: 2072-2079.

29. Seno Y, Shimada Y, Mizuguchi T, Tanikawa A, Horiguchi M. Compliance with the face-down positioning after vitrectomy and gas tamponade for rhegmatogenous retinal detachments. Retina. 2015; 35:1436-1440.
Clinical Ophthalmology

\section{Publish your work in this journal}

Clinical Ophthalmology is an international, peer-reviewed journal covering all subspecialties within ophthalmology. Key topics include: Optometry; Visual science; Pharmacology and drug therapy in eye diseases; Basic Sciences; Primary and Secondary eye care; Patient Safety and Quality of Care Improvements. This journal is indexed on

\section{Dovepress}

PubMed Central and CAS, and is the official journal of The Society of Clinical Ophthalmology (SCO). The manuscript management system is completely online and includes a very quick and fair peer-review system, which is all easy to use. Visit http://www.dovepress.com/ testimonials.php to read real quotes from published authors. 